\title{
The correlation between the cardiovascular instability and the size of the developed ischaemic lesions in patients who underwent carotid stenting
}

The Neuroradiology Journal 0 (0) 1-9

(C) The Author(s) 2021 Article reuse guidelines: sagepub.com/journals-permissions DOI: $10.1177 / 1971400920988667$ journals.sagepub.com/home/neu

@SAGE

\author{
Sándor Csizmadia ${ }^{1}$ (D), Zsófia Kaszás ${ }^{1}$, Róbert Klucsai ${ }^{2}$, Éva Bartha ${ }^{2}$ and \\ Erika Vörös ${ }^{2}$
}

\begin{abstract}
Aims: In this study we investigated the relationship between cardiovascular instability and the size of the developed ischaemic lesions during carotid stent implantation by diffusion-weighted (DWI) magnetic resonance (MR) examination. Material and methods: We retrospectively analyzed anaesthesia reports and follow-up MR examinations after stent implantation of 192 patients in a 3-year period. Nineteen aspects of cardiovascular status were analyzed. We registered the duration of the intervention, low and high blood pressure (BP) values during anaesthesia and heart rates. The fluctuations of BP and heart rate and the time of their compensations after the stent expansion were also recorded. Values were compared with the number and the size of ischaemic lesions on DWI scans. We used Spearman and Kendall rank correlations and Welch's tests for statistical analysis. Values of $p \leq 0.05$ were considered as statistically significant.

Results: Decreased heart rate significantly correlated with the number $(p=0.0123)$ and size $(p=0.00323)$ of ischaemic lesions during stent expansion. Other cardiovascular parameters did not show any significant correlations.

Conclusions: Our results indicate that only heart rate attenuation affects the size of ischaemic lesions; thus the prevention of bradycardia is highly important.
\end{abstract}

\section{Keywords}

Carotid stent implantation, microembolization, hypoperfusion, diffusion-weighted magnetic resonance imaging, bradycardia

\section{Introduction}

Atherosclerosis leads to increased mortality and morbidity in the Western world. Morphologically, the atheromatous intimal plaque is pathological. It is a well-circumscribed lesion that bulges into the lumen, the cut surface of which is yellowish-white. Symptoms are the result of complications caused by the plaque. The structure of the plaques is important in the evolution of symptoms. Instable, vulnerable plaques have a greater lipid core than the covering fibrous cap. In these cases, haemodynamic changes can lead to the rupture of these plaques easily. The stable plaque grows slowly for several years, gradually bulging into the lumen and progressively decreasing the amount of circulating blood in the stenotic lumen. ${ }^{1}$

Atherosclerosis can damage cervical blood vessels too. The symptoms of carotid stenosis are broad, and it can also be asymptomatic. It is considered as symptomatic when transitory ischaemic attack (TIA), amaurosis fugax or ischaemic stroke has occurred on the ipsilateral side of the stenosis in the last 6 months and neurological symptoms originate from carotid stenosis. $^{2}$

There are several ways to treat carotid stenosis besides medications. Carotid angioplasty, the minimally invasive, interventional procedure, competes with carotid endarterectomy as the oldest method. Each method has its advantages and disadvantages. During percutaneous transluminal angioplasty, a fluoroscopyguided catheter is inserted into the carotid artery. On reaching the stenosis, a balloon is inflated and a stent is deployed to achieve the correct size of the lumen. ${ }^{3-5}$

Microembolization and hypoperfusion during the procedure are well-known adverse events accompanying

\footnotetext{
${ }^{1}$ Affidea Hungary Ltd. Budapest, Hungary

${ }^{2}$ Department of Radiology, University of Szeged, Hungary

Corresponding author:

Sándor Csizmadia, Szivárvány utca 23/A, Hódmezővásárhely H-6800, Hungary.

Email: csizmadia.sandor.89@gmail.com
} 
carotid stent implantation that may cause ischaemic injury of the brain. Microembolization means that small parts of the plaque are detached and may occlude vessels distally. Any mechanical trigger on the plaques such as contact by a catheter, balloon dilatation or stent implantation can detach particles from them. Ischaemic lesions developing this way are rarely clinically symptomatic, but they are traceable by magnetic resonance (MR) examination. It must be stressed that late embolization is also an important complication and may lead to ischaemic injuries as well.,

Bradycardia and hypotonia often occur during balloon catheter dilatation and stent implantation. They may result in brain hypoperfusion through the baroreceptors of the carotid system and its reflexes. At the bifurcation of the common carotid artery, carotid sinus and glomus caroticum can be found. Both areas are rich in baroreceptors. These receptors are sensitive to mechanical triggers such as the tension of the blood vessel's wall. Mechanoreceptors affect the heart chronotropically and dromotropically via the fibres of the vagus nerve and the thalamus. In the event of hypertension baroreceptor reflex decreases blood pressure (BP) by mitigating heart rate and total peripheral resistance. $^{8-10}$

To sum up, invasive treatment of carotid stenosis can provoke cerebrovascular complications. However, therapy is essential since atheromatous plaques and stenosis are potentially harmful. The risks and benefits of the intervention should always be considered.

The aim of our retrospective study was to find correlation between the developed cardiovascular instability and the size and number of ischaemic lesions, both during the whole interventional procedure and immediately after carotid stent implantation using diffusionweighted (DWI) MR examination. ${ }^{11,12}$

\section{Methods and materials}

\section{Patients and intervention protocol}

The medical records of 192 patients were examined retrospectively in the Department of Radiology at the University of Szeged. The patients received carotid stents between 30 June 2014 and 17 July 2017. We analyzed plaques with the help of the previously obtained carotid computed tomography (CT) angiogram. We did not perform an intervention if the density of the plaque was less than 60 Hounsfield units (HU) or we found ulcerated plaque or concentric calcified plaque. The degree of the stenosis was between $75 \%$ and $90 \%$. Interventions were performed under local anaesthesia with anaesthesiologist supervision. During the procedure the common femoral artery was punctured, the catheter was guided to the carotid stenosis, the balloon dilatation was performed and the stent was implanted thereafter. Wallstent (Boston Scientific, Marlborough, MA, USA) was implanted in every case. A embolic protection device was not used. All of the interventions were performed by an expert radiologist. The operator had special licence for neuroradiology interventions. After the interventions, the patients were observed closely for 48 hours. The procedure is depicted in Figure 1 showing the status before and after balloon dilatation with the implanted stent. An MR scan was performed 24 hours after the procedure. Circulatory parameters during the interventions were collected first. Systolic and diastolic BP values, heart rate, respiratory rate and oxygen saturation in every minute were collected from the medical records of anaesthesiologists. The developed ischaemic areas were detected using DWI MR scans.

\section{Examined cardiovascular parameters}

For every intervention two different statuses were investigated according to the reports. Firstly, cardiovascular parameters and their changes during the entire procedure were registered. Additionally, changes of cardiovascular parameters at the moment of the stent dilatation were recorded for all patients. The start and the end point were recorded to measure the whole duration of the procedures. We noted every case where hypotension occurred; furthermore, we recorded the minimum value and the duration of the periods of hypotension. Hypotension was defined as systolic BP lower than $100 \mathrm{mmHg}$ or diastolic BP lower than $60 \mathrm{mmHg}$. We noted every case where hypertension occurred and recorded its duration and the highest $\mathrm{BP}$ value. In the case of hypertension, we divided the values into two subgroups depending on whether the systolic BP was over $140 \mathrm{mmHg}$ or $160 \mathrm{mmHg}$. The duration and the lowest heart rate of bradycardia were also recorded. The durations of tachycardia with the highest heart rate were noted too. Bradycardia was determined as less than 60 beats per minute (bpm), tachycardia as more than $100 \mathrm{bpm}$. If the $\mathrm{BP}$ or heart rate changed, we summarized the periods with the abnormal values and this was used for calculations.

We also examined numerous cardiovascular parameters at the moment of the stent dilatation. The time interval between the starting point of the intervention and the point of the balloon dilatation was registered in every case. We registered the systolic and diastolic values of the BP immediately before and after stent implantation. We recorded the heart rate right before and after stent implantation also. We used the same border values given above. The starting point of cardiovascular instability at dilatation and the point at which the values returned to normal again, i.e. compensation, were recorded. Compensation time was determined as the period between them. If the values were not normalized during the intervention until the end of the procedure, we used the end point value as the end of the compensation. Where we noticed hypotension or bradycardia after the dilatation, we recorded the time that was needed for the registered parameters 


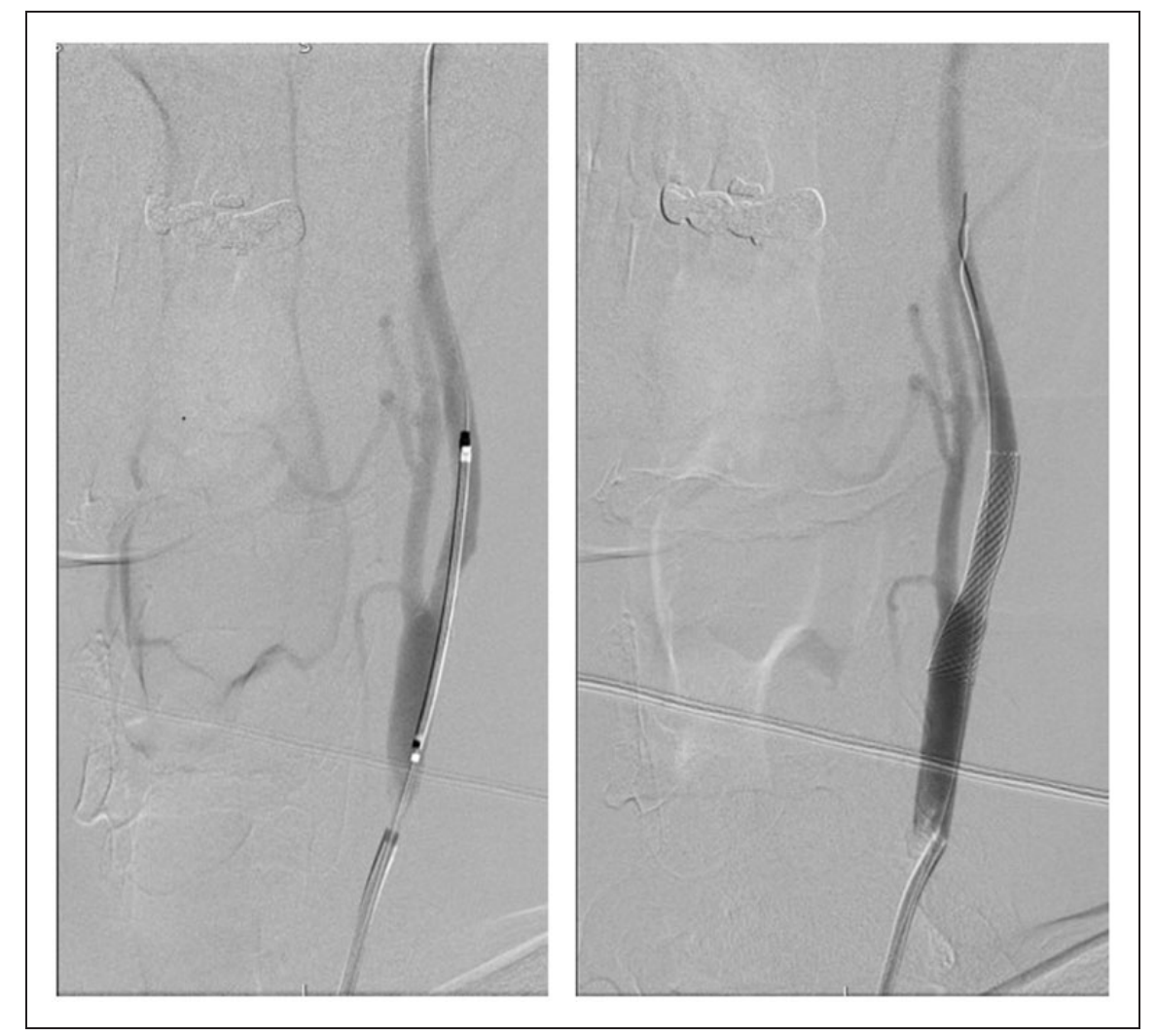

Figure 1. Digital subtraction angiography image of a 52-year-old male patient who received stent implantation on the left side.

to reset to normal values. Figure 2 shows the cardiovascular parameters in an anaesthesiologist's record.

\section{Determining ischaemic lesions}

All of the interventions were scheduled. The patients had no signs of acute stroke at the time of the procedure; therefore, we did not perform preoperative MR examinations. Twenty-four hours after the procedure we performed DWI and fluid-attenuated inversion recovery (FLAIR) sequences MR examinations. We used a $1.5 \mathrm{~T}$ Signa Excite MRI machine (General Electric Medical Systems, Fairfield, CT, USA). Areas with diffusion restriction were identified. The number, size, the side and the location (supra- or infratentorial) of these areas were noted. Images were analyzed by two independent radiologists. Figure 3 demonstrates ischaemic lesions on a DWI scan.

We compared the two-measurement series with each other to detect any differences regarding the number of diffusion-restricted areas. If a difference occurred, the images were revised. Apparent diffusion coefficient (ADC) values were measured in all of the questionable cases. If the ADC value of the lesion was less than 0.007 , it was considered as a real diffusion restriction. Figure 4 shows the ADC value measurement procedure. Extension was determined by the usage of antero-posterior (AP) and latero-lateral (LL) diameters. In this case the lesions were also measured by the two independent radiologists. The two measurements were averaged and then multiplied to get the areas used for calculations. If a lesion occurred in more than one slice, then the areas of the same lesions were added to get its approximate threedimensional size. These lesions were considered as one lesion regardless of the number of slices used to identify them.

\section{Patients and exclusion criteria}

Several patients were excluded because they were not appropriate for the aim of our study. In total, 37 patients were excluded because of inaccessible or incomplete anaesthesiologist's records; 29 patients were excluded because of motion or metal artefact during MR examinations and we could not analyse the images satisfactorily. Additionally, another seven patients were excluded because we identified cerebral media artery occlusion or carotid occlusion, or we performed further interventions like subclavian dilatation. One patient was excluded with statistical outlier values. Extended ischaemic injuries were found in three cases, and they were considered as outliers also. In total, 77 patients were excluded because of the abovementioned reasons.

Medical records including cardiovascular parameters for the whole procedure and the number size of ischaemic lesions were complete for 115 patients (55 female, 60 male). The average age was 67.3 years (range 47 to 87 years). In 45 cases the procedure 


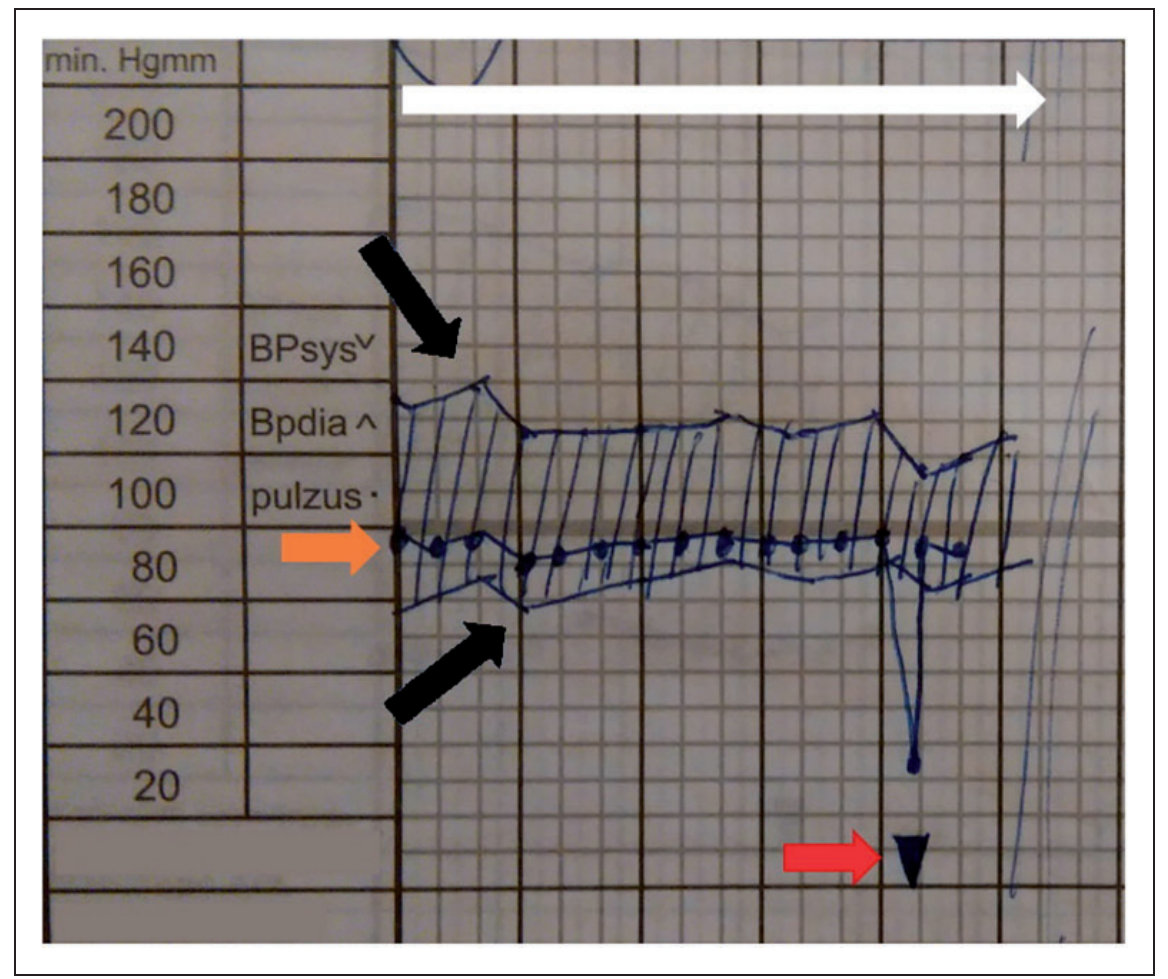

Figure 2. Cardiovascular parameters in an anaesthesiologist's record. Systolic and diastolic BP values (black arrows), heart rate (orange arrow) and the moment of balloon dilatation (red arrow) were recorded. Parameters are shown in timeline (white arrow) by the record.

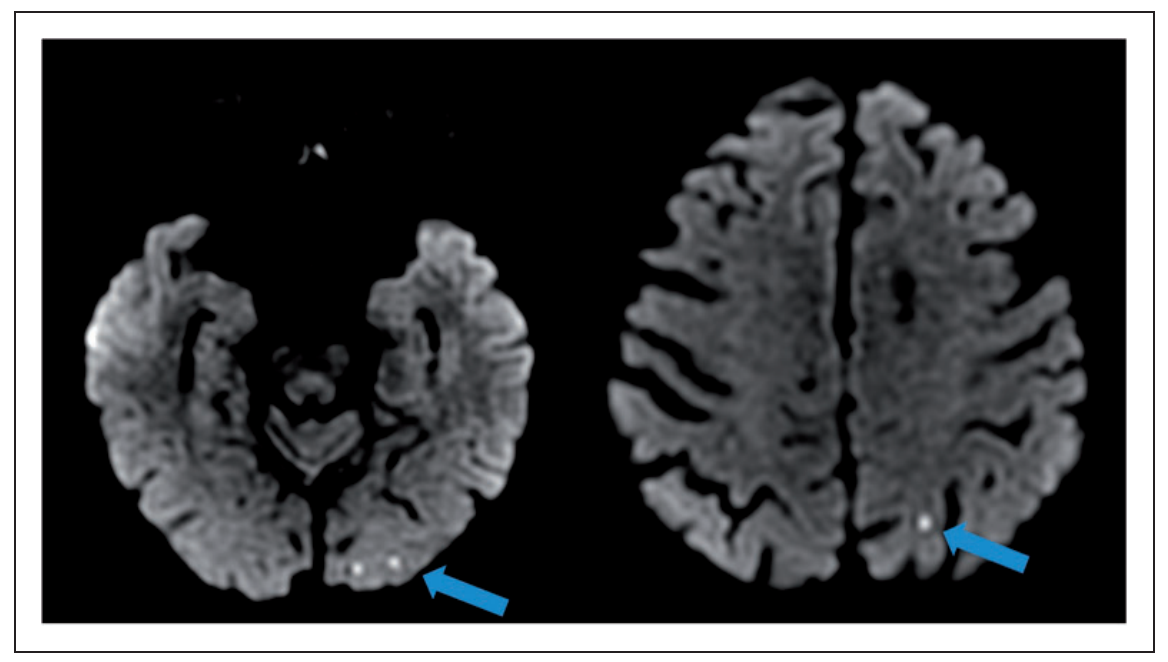

Figure 3. Diffusion-weighted magnetic resonance image of a 72-year-old male patient after stent implantation on the left side. Supratentorial ischaemic lesions are easily identifiable (blue arrows).

was performed on the right side and in 70 cases on the left side.

Additional patients were excluded because of missing values at the moment of dilatation. In 29 cases the exact time of dilatation was not recorded. In 86 cases the medical records of cardiovascular parameters at the moment of stent implantation and the number and size of ischaemic lesions was complete (44 female, 42 of male). The average age was 66.5 (range 47 to 87 years). Dilatation was performed on the right side in 38 cases and on the left side in 38 cases.

\section{Statistical analysis}

Data were analyzed by Microsoft Office ProPlus 365 Excel Analysis ToolPak and IBM SPSS Statistics Version 25 software. In total, 26 parameters were collected from anaesthesiologists' records and six parameters were collected from MR scans.

Non-parametric statistical tests were used since we hypothesized that the number and size of ischaemic lesions are not normally distributed. The following statistical tests, which do not require normal distribution of the statistical groups, were used: Spearman rank 


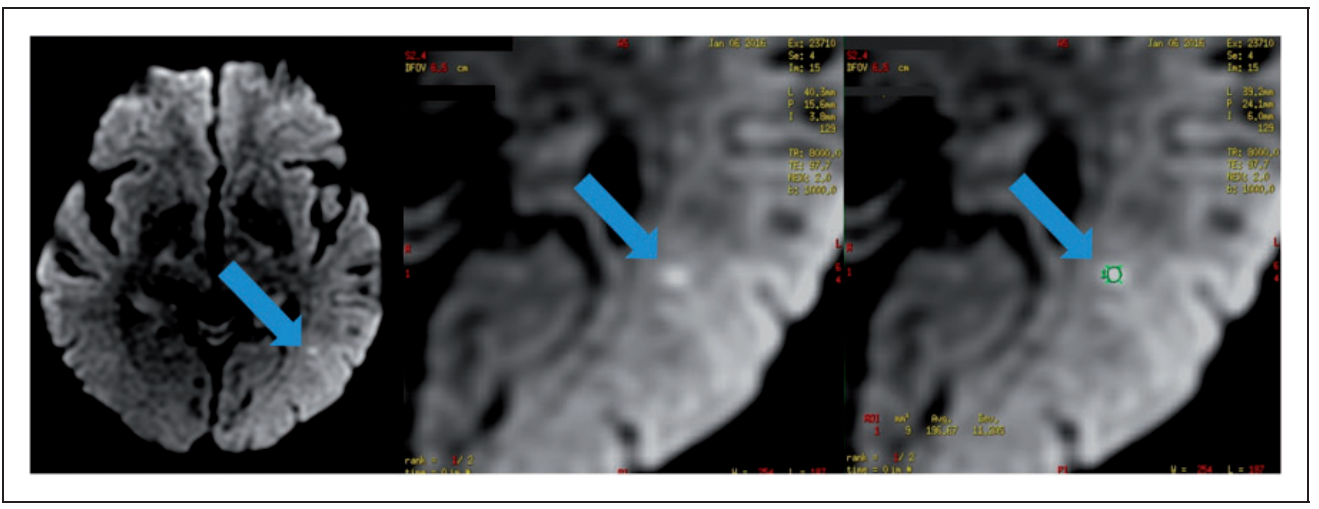

Figure 4. Ischaemic lesion on the left side after stent implantation in a 65-year-old female patient. Diffusion restriction was proved by apparent diffusion coefficient measurement.

correlation, Kendall rank correlation, Welch test, Pearson rank correlation. $p \leq 0.05$ was considered as statistically significant. Data were presented as mean \pm standard deviation.

\section{Results}

\section{Results of the entire procedure period}

Values of the cardiovascular parameters. In total, 115 patients' cardiovascular parameters were measured and averaged from the medical records of anaesthesiologists. The average time for the whole procedure was $55 \pm 19 \mathrm{~min}$ (the shortest was $35 \mathrm{~min}$, the longest 140 min). Hypotension was recorded in 26 cases, with the average time being $17 \pm 19 \mathrm{~min}$ (the shortest lasted for $35 \mathrm{~min}$, the longest $40 \mathrm{~min}$ ); the lowest value was 75/ $35 \mathrm{mmHg}$. Systolic values were over $140 \mathrm{mmHg}$ in 100 cases, the average time for which was $43 \pm 21 \mathrm{~min}$ (the shortest was $3 \mathrm{~min}$, the longest $140 \mathrm{~min}$ ); the highest value was $215 / 115 \mathrm{mmHg}$. Systolic values were over $160 \mathrm{mmHg}$ in 59 cases, the average time for which was $31 \pm 22 \mathrm{~min}$ (the shortest was $5 \mathrm{~min}$, the longest $95 \mathrm{~min}$ ). Bradycardia occurred in 55 cases, the average time for which was $14 \pm 19 \mathrm{~min}$ (the shortest was $1 \mathrm{~min}$, the longest $95 \mathrm{~min}$ ); the lowest heart rate was $10 \mathrm{bpm}$. Tachycardia occurred in six cases, the average time for which was $34 \pm 35 \mathrm{~min}$ (the shortest was $5 \mathrm{~min}$, the longest $100 \mathrm{~min}$ ); the highest value was $140 \mathrm{bpm}$.

Parameters of ischaemic lesions. Eighty-four out of 115 patients developed ischaemic lesions. In total, 469 lesions were analyzed. The average size was $3.43 \times 3.32 \mathrm{~mm} \quad(\mathrm{AP} \times \mathrm{LL})$. In 26 cases a lesion appeared in more than one slice because of its extension. Out of 469 lesions, 262 were on the left side and 207 were on the right side. In total, 462 diffusion restricted lesions were supratentorial, and seven of them were infratentorial.

Statistical results for the whole procedure period. All recorded cardiovascular parameters were correlated with the number and size of ischaemic lesion. Only one of the registered cardiovascular parameters did not correlate significantly. Table 1 summarises the statistical analysis and $p$-values. The presence of tachycardia, its period and its maximum values cannot be interpreted due to the low number of cases.

\section{Results at the moment of stent dilatation}

Values of the cardiovascular parameters. Right before dilatation the average systolic BP was $152 \pm 19 \mathrm{mmHg}$, the diastolic was $79 \pm 12 \mathrm{mmHg}$ and heart rate was $75 \pm 15$ bpm. Right after dilatation the average systolic BP was $134 \pm 24 \mathrm{mmHg}$, the diastolic was $72 \pm 15 \mathrm{mmHg}$ and heart rate was $61 \pm 22 \mathrm{bpm}$. Out of 86 cases 15 patients developed hypotension; the shortest compensation time was $5 \mathrm{~min}$, the longest compensation time was $25 \mathrm{~min}$ and the average compensation time was $11 \pm 6$ min. Bradycardia occurred in 37 cases; its shortest compensation time was $2 \mathrm{~min}$, the longest compensation time was $60 \mathrm{~min}$ and the average compensation time was $8 \pm 10 \mathrm{~min}$. Cardiovascular instability occurred in 44 cases, and in eight cases both bradycardia and hypotension occurred.

Parameters of ischaemic lesions. Sixty-one out of 86 patients had ischaemic lesions on MR scans after the procedure. In total, 333 diffusion-restricted lesions in 61 patients were registered. Twenty-six lesions extended into more than one slice because of their size. In total, 330 lesions were supratentorial and three lesions were located infratentorially; 170 lesions were on the right side and 163 were on the left side. The average dimension was $3.58 \times 3.49 \mathrm{~mm}(\mathrm{AP} \times \mathrm{LL})$.

\section{Statistical results at the moment of stent dilatation}

There was no significant correlation between the duration of the procedure (starting time of the procedure and time of the dilatation) and the examined parameters of the ischaemic lesions. We used Kendall rank correlation for statistical analysis for number of lesions $(p=0.3402)$ and their size $(p=0.4792)$. 
Table 1. Correlation of cardiovascular parameters and the number and extension of ischaemic values.

\begin{tabular}{llll}
\hline Registered parameters & Statistical probe & Number of lesions & Size of lesions \\
\hline Duration of the whole procedure & Kendall rank correlation & $p=0.5116$ & $p=0.5369$ \\
Presence of hypotension & Welch test & $p=0.7042$ & $p=0.4445$ \\
Period of hypotension & Kendall rank correlation & $p=0.9543$ & $p=0.8438$ \\
Minimum value of hypotension & Kendall rank correlation & $p=0.8139$ & $p=0.3054$ \\
Presence of BP over $140 \mathrm{mmHg}$ & Welch test & $p=0.6344$ & $p=0.5093$ \\
Period of BP over $140 \mathrm{mmHg}$ & Kendall rank correlation & $p=0.2217$ & $p=0.2221$ \\
Maximum value of BP over $140 \mathrm{mmHg}$ & Kendall rank correlation & $p=0.3846$ & $p=0.4632$ \\
Presence of BP over $160 \mathrm{mmHg}$ & Welch test & $p=0.0589$ & $p=0.0608$ \\
Period of BP over $160 \mathrm{mmHg}$ & Kendall rank correlation & $p=0.2399$ & $p=0.3539$ \\
Maximum value of BP over $160 \mathrm{mmHg}$ & Kendall rank correlation & $p=0.8999$ & $p=0.6734$ \\
Presence of bradycardia & Welch test & $p=0.8981$ & $p=0.1147$ \\
Period of bradycardia & Kendall rank correlation & $p=0.9872$ & $p=0.5281$ \\
Minimum value of bradycardia & Kendall rank correlation & $p=0.2985$ & $p=0.3042$ \\
Presence, period and maximum value of tachycardia & Not applicable & & \\
\hline
\end{tabular}

BP: blood pressure.

Statistically, we got more reliable results when the parameters of the ischaemic lesions were correlated with systolic and diastolic BP changes during dilatation compared to the usage of correlation with systolic and diastolic BP values before and after the dilatation. The average systolic BP decrease was $18.6 \pm 23.95 \mathrm{mmHg}$ and the diastolic BP decrease was $7.73 \pm 13.83 \mathrm{mmHg}$ during the dilatation. There was no significant correlation (Kendall rank correlation) between the attenuation of systolic BP and the number of lesions $(p=0.287)$ and their size $(p=0.4661)$. We also could not prove a significant difference between the attenuation of diastolic BP and the number $(p=0.2832)$ and size $(p=0.3956)$ of the lesions. We used the heart rate decline during the dilatation for the statistical analysis, the average value for which was $14 \pm 20.58 \mathrm{bpm}$. This alteration correlated significantly with the number of lesions $(p=0.0123)$ and their size $(p=0.00323)$ by Kendall rank correlation.

The compensation time of bradycardia did not correlate significantly with the lesion number $(p=0.06472)$ and size $(p=0.2744)$ by Kendall rank correlation. The $p$-value of the number of ischaemic lesions is approaching the point of $p \leq 0.05$ and can indicate a connection between the two variables. The correlation between the compensation time of hypotension and ischaemic lesions could not be interpreted due to the low case number and the outlier values. Generally, BP decreased after dilatation, but in most cases it did not decrease below $100 \mathrm{mmHg}$ systolic or $60 \mathrm{mmHg}$ diastolic value.

\section{Discussion}

\section{Correlation between embolization and cardiovascular instability}

Carotid stent implantation is accompanied by microembolization and hypoperfusion, which can lead to ischaemic brain injury. In serious cases stroke may develop. Microembolization means that small particles of the plaques are detached and can reach the brain via circulation during and after the procedure. ${ }^{13,14}$

Several studies demonstrated that ischaemic brain injury after stent implantation cannot be caused by microembolization exclusively. At the moment of dilatation hypotonia and brain hypoperfusion caused by cardiovascular instability can increase the chances of MR detectable ischaemic brain lesion development. ${ }^{15-17}$

It is already proven that carotid stent implantation increases cardiovascular instability, and the baroreceptor reflex plays a major role in the evolving hypotonia. Whether hypo- or hypertension occurs, turbulent blood flow increases the mechanical effects of cardiac instability to the injured blood vessel wall. The two processes potentiate the effect of each other. Several studies have investigated haemodynamic instability emphasizing the importance of the postoperative period. On the contrary just a few studies have examined the effect of intraprocedural cardiac instability. ${ }^{18-20}$

Our study demonstrates that balloon catheter dilatation with stent implantation and the cardiac instability during the whole procedure do not significantly increase the number of MR detectable ischaemic lesions and extension 24 hours after the procedure. Only patients with over $160 \mathrm{mmHg}$ systolic BP approached the level of significance. This suggest that extremely high BP during the procedure can have an influence on the development of ischaemic lesions. Regarding this, further investigations are required. On analysis of our data, we did not find any significant correlation among the size of periprocedural ischaemic brain lesions, the amount of time till dilatation, the duration of the whole intervention and the systolic and diastolic BP changes during the procedure. Therefore, the attenuation of systolic and diastolic BP does not mean there is an additional risk factor for ischaemic brain lesions, although in extreme cases 
it is necessary to correct them to maintain the integrity of the circulation. Heart rate attenuation during the dilatation procedure led to significant correlation with the ischaemic lesions' number and size. There was no significant correlation between the compensation time of bradycardia and the number of ischaemic lesions, but the $p$-value of 0.065 highly suggests a connection between them.

Cardiovascular instability during dilatation can be explained with the baroreceptor reflex. Activation of it has negative chronotropic and dromotropic effect via the parasympathetic nervous system. It is a simple idea to prevent or treat bradycardia and hypotonia with atropine. $^{21,22}$

Atropine is a parasympatholytic drug, and it can be classified as an anticholinergic agent in other aspects. At the parasympathetic end plates, it inhibits impulse conduction competitively by decreasing the parasympathetic tone. Atropine $0.5-1 \mathrm{mg}$ is used to prevent or treat bradycardia and hypotonia during carotid stenting. ${ }^{23}$

Several studies have investigated whether atropine significantly decreases the incidence of hypotonia and bradycardia during the procedure. Cayne et al. demonstrated that receiving atropine as prevention before dilatation significantly decreases the incidence of hypotonia and bradycardia after dilatation. ${ }^{24}$ On the other hand, Qureshi et al. demonstrated that receiving atropine as prevention paradoxically significantly increased the chance of postoperative bradycardia. $^{25}$ This study recommends caution when using atropine. The Stenting versus Endarterectomy for Treatment of Carotid Artery Stenosis study ${ }^{7}$ suggests a protocol of $1 \mathrm{mg}$ of atropine intravenously prophylactically.

According to our study, bradycardia must be compensated and larger heart rate attenuation must be prevented or corrected as soon as possible to avoid the evolution of ischaemic lesions. We cannot conclude the same regarding BP parameters, but it is important to maintain cardiovascular integrity with proper anaesthesia during the procedure.

\section{Limitations and additional influencing factors of embolus formation}

For the objectivity we must consider the limitations of our study. We disregarded patients' previous clinical history. Previous symptoms (stroke or TIA), comorbidity and risk factors (smoking, body weight, lifestyle) in particular might be important. We documented the grade of stenosis and we also performed plaque analysis before the procedure with the help of the previously obtained CT angiography. Although these aspects are important and influence the extension of developed ischaemic lesions, previous studies have already examined them. Therefore, we did not include these parameters in the statistical tests.

Biasi GM et al. investigated whether preprocedural plaque analysis can provide more precise risk analysis for periprocedural stroke. ${ }^{26}$ This study showed that hypoechogenic, soft plaques are associated with higher risk for stroke, and therefore they predispose to stroke. Additional studies indicate correlations among atherosclerotic plaque structure, plaque rupture and consecutive ischaemic brain lesions. Plaque morphology is one of the most important factors in post-procedural stroke, but this has already been proven. ${ }^{10,27}$

The structure of the plaque is not just significant in embolization, but it also has an important role in the development of cardiovascular instability. Gupta et al. demonstrated that calcified plaques predispose individuals to cardiovascular instability. ${ }^{20}$ Owing to their stable structures, the force of dilatation mediates more effectively to the baroreceptors, thus activating the baroreceptor reflex. Several studies proved that the incidence of cardiovascular instability is strongly correlated with the location of the stenosis. Stent implantation close to the carotid sinus significantly increased cardiovascular instability compared to more distant sites. ${ }^{20}$

Currently, there is no generally accepted guideline for proper stent selection. We implanted open Wallstents; therefore, the type of stent has no effect on our data. Csobay-Novák et al. proposed recommendations for the usage of different stent types. ${ }^{28}$ They have also demonstrated that cardiovascular instability occurs more frequently with implantation of shorter stents. It can be explained that shorter stents have stronger radial force to the narrowed blood vessel wall. Stronger radial force means greater transmural force stimulating the carotid system's own receptors, thus increasing cardiovascular instability. Similarly, a significant correlation has been proved between circulatory fluctuation and the grade of dilatation (difference in the lumen diameters before and after the procedure). ${ }^{21,29-31}$

As outlined above, it is obvious that many studies have already examined carotid stenting, but several aspects of this procedure are still unclear. In our study we aimed to focus on some of those unanswered questions, and we should consider broadening our treatise by measuring other parameters.

An additional limitation of our study was that we did not investigate patients' medication. According to other studies, this is an important parameter regarding the prevention and therapy of bradycardia. Medications during anaesthesia are included in the anaesthesiologists' record. We have been analysing them, and it will be the subject of our next study.

Tools which can prevent distal embolization are still an undiscovered area. Several physicians use these tools, but they cannot give full protection, especially against prolonged embolization, and their insertion can lead to embolism. ${ }^{32,33}$ We did not use embolic protection devises in any of the procedures because of the additional risk. Thus, we could not investigate their effect. 


\section{Conclusions}

To summarize, we can conclude that cardiovascular instability and periprocedural embolization caused ischaemic lesions. They are technically connected to carotid stent implantations and occur frequently. Our results suggest that ischaemic lesions and carotid stent implantations are not independent from each other.

Heart rate attenuation during stent dilatation and the number and size of ischaemic brain lesions developed within 24 hours and showed unequivocal correlation. Our study proved clearly that bradycardia should be prevented or ceased quickly to save brain tissue. Most of the lesions are asymptomatic but can still cause the death of millions of neurons, which cannot be disregarded.

Despite our results, stent implantation can still be considered safe since intraoperative bradycardia is preventable or treatable by proper anaesthesia. According to our results cardiovascular instability and postprocedural ischaemic lesions can accompany stent implantation. These effects are a technicality of the intervention and should be considered at surgical planning, but they do not mean additional risk.

\section{Conflict of interest}

The authors declared no potential conflicts of interest with respect to the research, authorship, and/or publication of this article.

\section{Funding}

The author(s) received no financial support for the research, authorship, and/or publication of this article.

\section{ORCID iD}

Sándor Csizmadia (D) https://orcid.org/0000-0002-2153-4293

\section{References}

1. Robbins SL. Basic pathology. 9th ed. Amsterdam: Elsevier, 2013, pp. 335-344.

2. Petty GW, Brown RD Jr, Whisnant JP, et al. Ischemic stroke subtypes: a population-based study of incidence and risk factors. Stroke 1999; 30: 2513-2516.

3. Massara M, Notarstefano S, Gerardi P, et al. Unstable atherosclerotic plaque in the common carotid artery: Diagnosis and treatment strategy. Semin Vasc Surg 2018; 31: 88-90.

4. Roffi M, Yadav JS. Carotid stenting. Circulation 2006; 114: 1-5.

5. Moran CJ, Cross DT, Derdeyn CP. Techniques of carotid angioplasty and stenting. Neuroimaging Clin $\mathrm{N} \mathrm{Am}$ 2007; 17: 337-353.

6. Gerard P, Stansby SM. Practical carotid artery stenting. Berlin: Springer, 2009, pp. 143-158.

7. Caputo R, et al. Stenting versus endarterectomy for treatment of carotid-artery stenosis. $N$ Engl J Med 2010; 363: 1663-1674.

8. Andani R, Khan YS. Anatomy, head and neck, carotid sinus. StatPearls Publishing, https://www.ncbi.nlm.nih.
gov/books/NBK554378/ (2020, accessed 19 February 2020).

9. Armstrong M, Moore RA. Physiology, baroreceptors. StatPearls Publishing, https://www.ncbi.nlm.nih.gov/ books/NBK538172/ (2020, accessed 19 April 2020).

10. Bonati LH et al. New ischaemic brain lesions on MRI after stenting or endarterectomy for symptomatic carotid stenosis: a substudy of the International Carotid Stenting Study (ICSS). Lancet Neurol 2010; 9: 353-362.

11. Altinbas A, Algra A, Bonati LH, et al. Periprocedural hemodynamic depression is associated with a higher number of new ischemic brain lesions after stenting in the international carotid stenting study-MRI substudy. Stroke 2014; 45: 146-151.

12. Doig D. et al. Carotid anatomy does not predict the risk of new ischaemic brain lesions on diffusion-weighted imaging after carotid artery stenting in the ICSS-MRI substudy. Eur J Vasc Endovasc Surg 2016; 51: 14-20.

13. Gossetti B, Gattuso R, Irace L, et al. Embolism to the brain during carotid stenting and surgery. Acta Chir Belg 2007; 107: 151-154.

14. Orlandi G, Fanucchi S, Fioretti C, et al. Characteristics of cerebral microembolism during carotid stenting and angioplasty alone. Arch Neurol 2001; 58: 1410-1413.

15. Leisch F, Kerschner K, Hofmann R, et al. Carotid sinus reactions during carotid artery stenting: Predictors, incidence, and influence on clinical outcome. Catheter Cardiovasc Interv 2003; 58: 516-523.

16. Pappadà G, Beghi E, Marina R, et al. Hemodynamic instability after extracranial carotid stenting. Acta Neurochirurgica 2006; 148: 639-645.

17. Caplan LR, Ka SW, Gao S, et al. Is hypoperfusion an important cause of strokes? If so, how? Cerebrovasc Dis 2006; 21: 145-153.

18. Kojuri J, Ostovan MA, Zamiri N, et al. Hemodynamic instability following carotid artery stenting. Neurosurg Focus 2011; 30: 6-12.

19. Altinbas A, Algra A, Brown MM, et al. Effects of carotid endarterectomy or stenting on hemodynamic complications in the International Carotid Stenting Study: A randomized comparison. Int J Stroke 2014; 9: 284-290.

20. Gupta R, Abou-Chebl A, Bajzer CT, et al. Rate, predictors, and consequences of hemodynamic depression after carotid artery stenting. J Am Coll Cardiol 2006; 47: $1538-1543$.

21. Lavoie P, Rutledge J, Dawoud MA, et al. Predictors and timing of hypotension and bradycardia after carotid artery stenting. AJNR Am J Neuroradiol 2008; 29: 1942-1947.

22. Mlekusch W, Schillinger M, Sabeti S, et al. Hypotension and bradycardia after elective carotid stenting: frequency and risk factors. $J$ Endovasc Ther 2003; 10: 851-859.

23. Katzung BG, Masters SB, Trevor AJ. Basic \& clinical pharmacology. 12th ed. New York: McGraw-Hill Education, 2012, pp. 115-124.

24. Cayne NS, Faries PL, Trocciola SM, et al. Carotid angioplasty and stent-induced bradycardia and hypotension: Impact of prophylactic atropine administration and prior carotid endarterectomy. J Vasc Surg 2005; 41: 956-961.

25. Qureshi IA, Luft AR, Sharma M, et al. Frequency and determinants of postprocedural hemodynamic instability after carotid angioplasty and stenting. Stroke 1999; 30: 2086-2093. 
26. Biasi GM, Froio A, Diethrich EB, et al. Carotid plaque echolucency increases the risk of stroke in carotid stenting: The imaging in carotid angioplasty and risk of stroke (ICAROS) study. Circulation 2004; 110: 756-762.

27. Redgrave JNE, Lovett JK, Gallagher PJ, et al. Histological assessment of 526 symptomatic carotid plaques in relation to the nature and timing of ischemic symptoms: The Oxford plaque study. Circulation 2006; 113: $2320-2328$

28. Csobay-Novák C, Bárány T, Zima E, et al. Role of stent selection in the incidence of persisting hemodynamic depression after carotid artery stenting. $J$ Endovasc Ther 2015; 22: 122-129.

29. Ahn SH, Prince EA, Dubel GJ. Carotid artery stenting: Review of technique and update of recent literature. Semin Intervent Radiol 2013; 30: 288-296.
30. Müller-Hülsbeck S, Schäfer PJ, Charalambous N, et al. Comparison of carotid stents: An in-vitro experiment focusing on stent design. J Endovasc Ther 2009; 16: 168-177.

31. Ackerstaff RGA, et al. Prediction of early cerebral outcome by transcranial Doppler monitoring in carotid bifurcation angioplasty and stenting. J Vasc Surg 2005; 41: 618-624.

32. Chen CI, Iguchi Y, Garami Z, et al. Analysis of emboli during carotid stenting with distal protection device. Cerebrovasc Dis 2006; 21: 223-228.

33. Stojanov D, Ilic M, Bosnjakovic P, et al. New ischemic brain lesions on diffusion-weighted MRI after carotid artery stenting with filter protection: Frequency and relationship with plaque morphology. AJNR Am J Neuroradiol 2012; 33: 708-714. 